\title{
Parental role modelling and fruits and vegetables intake in European preschoolers: ToyBox-study
}

\author{
Pilar De Miguel-Etayo ${ }^{1,2}$, Paloma Flores $^{3}$, Javier Santabarbara ${ }^{4}$, Iris Iglesia $^{5}$, Greet Cardon ${ }^{6}$, \\ Violeta Iotova ${ }^{7}$, Berthold Koletzko ${ }^{8}$, Odysseas Androutsos ${ }^{9}$, Aneta Kotowska ${ }^{10}$, Yannis Manios ${ }^{9}$, \\ J. M. M. (Mai) Chin A Paw ${ }^{2}$ and Luis A Moreno ${ }^{1,12}$ \\ ${ }^{1}$ Growth, Exercise, NUtrition and Development (GENUD) Research Group. Instituto Agroalimentario de Aragón \\ (IA2), Instituto de Investigación Sanitaria Aragón (IIS Aragón). CIBER Fisiopatología de la Obesidad y Nutrición \\ (CIBERobn), Instituto de Salud Carlos III. University of Zaragoza, Zaragoza, Spain, \\ ${ }^{2}$ Child Health \& Care Research. VU University Medical Centre, Amsterdam, Netherlands, \\ ${ }^{3} 1$ Growth, Exercise, NUtrition and Development (GENUD) Research Group. Instituto Agroalimentario de Aragón \\ (IA2), Instituto de Investigación Sanitaria Aragón (IIS Aragón). CIBER Fisiopatología de la Obesidad y Nutrición \\ (CIBERobn), Instituto de Salud Carlos III. University of Zaragoza, Zaragoza, Spain, \\ ${ }^{4}$ Department of Microbiology, Preventive Medicine and Public Health, University of Zaragoza, Zaragoza, Spain, \\ ${ }^{5}$ Growth, Exercise, NUtrition and Development (GENUD) Research Group. Instituto Agroalimentario de Aragón \\ (IA2),Fundación Instituto de Investigación Sanitaria Aragón (IIS Aragón) y Red de Salud Materno-Infantil \\ (SAMID), Universidad de Zaragoza, Zaragoza, Spain, \\ ${ }^{6}$ Department of Movement and Sports Sciences, Ghent University, Ghent, Belgium, \\ ${ }^{7}$ Medical University Varna, Varna, Bulgaria, \\ ${ }^{8}$ Dr von Hauner Children's Hospital, University of Munich Medical Centre, Munich, Germany, \\ ${ }^{9}$ Department of Nutrition and Dietetics, School of Health Science and Education, Harokopio University, Athens, Greece, \\ ${ }^{10}$ The Children's Memorial Health Institute, Warsaw, Poland, \\ ${ }^{11}$ Growth, Exercise, NUtrition and Development (GENUD) Research Group. Instituto Agroalimentario de Aragón \\ (IA2), Instituto de Investigación Sanitaria Aragón (IIS Aragón). CIBER Fisiopatología de la Obesidad y Nutrición \\ (CIBERobn), Instituto de Salud Carlos III., Zaragoza, Spain and \\ ${ }^{12}$ Department of fisiology and nursing. Faculty of health science. University of Zaragoza, Zaragoza, Spain
}

\section{Abstract}

Introduction: Childhood obesity is rising in all countries. Dietary habits are modifiable factors which develop early in life. During growth, several factors, such as peer- influence and food availability, determine the development of food preferences and eating behaviour. Parents play also a key role model by influencing their own food intake.

Objetives: The purpose of this study was to assess the influence of parental role modelling, as predictor of fruits and vegetables intake in European pre-schoolers.

Methods: The present study included a sample of 6633 preschool children (51.9\% boys) from six European centres (Belgium, Bulgaria, Germany, Greece, Poland and Spain), 3.5 to 5.5 years of age, participating in the ToyBox-study. Data on parental role modelling related with their own fruits and vegetables intake (portions/day) and children's fruits and vegetables intake (portions/day) were collected via standardized proxy-administered questionnaires. Linear regression model was used to assess this association. The adjusted model included socioeconomic status and weight status.

Results: In the unadjusted model, boys whose parents consumed fruits, showed a mean intake of 0.09 (95\% CI: $0.08-0.11$; p $\leq 0.001)$ portions of fruits more than the boys whose parents did not consume fruits. Also, girls whose parents consumed fruits, had intake of $0.10(95 \%$ CI: $0.08-0.12 ; \mathrm{p} \leq 0.001)$ portions of fruits more than the girls whose parents did not consume fruits.

Moreover, boys whose parents consumed vegetables, showed a mean intake of $0.09(95 \% \mathrm{CI}: 0.07-0.11$; $\mathrm{p} \leq 0.001)$ portions of vegetables more than the boys whose parents did not consume vegetables. Also, girls whose parents consumed fruits, had intake of 0.11 (95\% CI: $0.09-0.13 ; p \leq 0.001)$ portions of vegetables more than the girls whose parents did not consume vegetables.

Finally, parental role related with fruits consumption explained 19.3\% of fruits intake in European pre-schoolers and the $17.8 \%$ of vegetables intake in boys and $21.9 \%$ of vegetable intake in girls taking into consideration the potential effect of socioeconomic status of the family and the weight status of the children.

Conclusions: Parental role model of fruit intake has moderate effect on the pre-schooler's dietary intake. However, home environment characteristics such as family rules or availability and accessibility of foods should be considered as potential factors related to food intake in pre-schoolers.

\section{Conflict of Interest}

There is no conflict of interest 